\title{
Preface Issue 3-2014
}

\section{Hans-Christoph Grunau}

(C) Deutsche Mathematiker-Vereinigung and Springer-Verlag Berlin Heidelberg 2014

Is it possible to model human societies and to predict on this basis some characteristic features of their long term development? This question is addressed by F. Thomas Bruss's survey article entitled "Grenzen einer jeden Gesellschaft". The starting point are two widely acceptable hypotheses governing the decisions of human beings. The modeling is done by means of branching processes, which depend on the available resources and the chosen politics. The main result is an enclosure theorem which states that there are two extreme forms of societies (weakest-first and strongest-first) which build an envelope for all possible societies within the underlying model.

The obituary for Wolfgang Schwarz, written by Karl-Heinz Indlekofer, Lutz G. Lucht and Jörn Steuding, reviews some of his most important results. His œuvre is focussed on the boundary behaviour of power series, Tauberian theorems, sieve methods and, mainly, on arithmetic functions. His monograph "Arithmetical functions" with Jürgen Spilker is a standard reference in this field of number theory. The Deutsche Mathematiker-Vereinigung is grateful to Wolfgang Schwarz for his commitment, among other things he served as its chairman in 1986 and 1987.

In collaboration with the Zentralblatt für Mathematik, we have in each issue a contribution to the category "Classics Revisited". Here, Niels Jacob reviews again Lars Hörmander's encyclopedic series of four monographs on "The analysis of linear partial differential operators". This review describes in some detail the contents and the huge influence of these books of the 1962 Fields Medalist. It is, however, not intended to serve as a kind of obituary on Lars Hörmander, who died on November 25, 2012. Something of this type is for example Nicolas Lerner's “Tribute to Lars Hörmander",

H.-Ch. Grunau ( $\bigotimes)$

Institut für Analysis und Numerik, Fakultät für Mathematik, Otto-von-Guericke-Universität, Postfach 4120, 39016 Magdeburg, Germany

e-mail: hans-christoph.grunau@ovgu.de 
which appeared in the June 2013-EMS-Newsletter (No. 88) and is available online also at http://www.math.jussieu.fr/ lerner/.

The recent books, which are reviewed in the current issue, are concerned with "The Mathematics of Planet Earth", Markov numbers-a topic from diophantine equations, nonlinear stochastic partial differential equations, and the topology of algebraic curves. 